\title{
A 2-bit, 3.1 GS/s, band-pass DSM receiver for active antenna systems
}

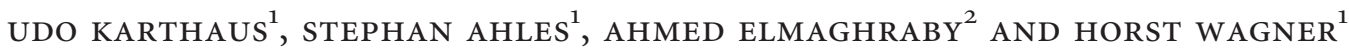

\begin{abstract}
This paper presents a radio frequency $(R F)$ continuous-time band-pass delta sigma modulator (CT BP DSM) receiver realized in a $180 \mathrm{~nm}$ SiGe BiCMOS technology. It also provides an introduction to active antenna systems (AAS) for cellular infrastructure base stations, which is the target application for this RF integrated circuit (IC). The internal quantizer and feedback digital to analog converter (DAC) resolution of the CT BP DSM is 2 bit. Without applying DAC linearization techniques such as trimming or dynamic element matching being utilized, measured performance parameters include an SNR and SNDR in $35 \mathrm{MHz}$ bandwidth of 56.7 and $53.7 \mathrm{~dB}$, respectively. IIP3 and noise figure are $-6.6 \mathrm{dBm}$ and $10 \mathrm{~dB}$, respectively. No image reception is noticeable within a measurement dynamic range of $83 \mathrm{~dB}$. When driven by single-carrier and three-carrier W-CDMA signals, adjacent channel leakage ratio (ACLR) is -62.6 and $-52.1 \mathrm{~dB}$, respectively, making the design also suitable as a modulator for a class-S power amplifier.
\end{abstract}

Keywords: Circuit design and applications, Active array antennas and components

Received 25 October 2012; Revised 05 March 2013; first published online 23 April 2013

\section{INTRODUCTION}

This paper first provides an introduction to active antenna systems (AAS) for cellular infrastructure base stations, which is the target application for the presented continuoustime band-pass delta sigma modulator (CT BP DSM). In addition, it outlines advantages and challenges of CT BP DSM receivers compared to other receiver architectures.

\section{A) System design: AAS versus traditional base stations}

Traditional cellular base stations employ passive antennas and remote radio heads (RRHs) with a single, high power amplifier, two low noise amplifiers (LNAs), and two coaxial feeder cables (one for each polarization) from the RRH to the passive antenna, and a passive feed network connecting the single RRH to typically 5-8 radiator elements inside the passive antenna. The feed network provides radio frequency $(\mathrm{RF})$ power combining for the receive (RX) signal and RF power splitting for transmit (TX) signals with appropriate phases and power ratios as required for shaping the antenna radiation pattern.

While active antennas have been used for a long time in applications such as radar, only recently AAS have gained increasing attention and interest of the cellular infrastructure

${ }^{1}$ Ubidyne GmbH, Magirusstr. 43, 89077 Ulm, Germany

${ }^{2}$ University of Erlangen-Nuremberg, Cauerstr. 9, 91058 Erlangen, Germany

Corresponding author:

U. Karthaus

Email: udo.karthaus@gmx.net market. Meanwhile, AAS are being promoted and announced by most suppliers of this industry, e.g. [1-3].

In contrast to RRHs with passive antennas, the distributed AAS as shown in Fig. 1 employs multiple transceivers inside the antenna housing, with two transceivers (one for each polarization) being directly connected to each antenna element. In the following, we will refer to such AAS systems that fulfill the aspect of a distributed transceiver architecture, not to the simple mechanical integration of an RRH into a passive antenna housing.

In such a distributed AAS, signal combining and splitting are done digitally, eliminating the need for physical RF power splitters, combiners, and (optional) phase shifters. Also, the costly, heavy, and lossy RF feeder cables are abandoned. The receive signals of $N$ transceivers are digitally combined in the central DSP (digital signal processing) with complex antenna coefficients $\mathrm{c}_{1} \mathrm{~A}, \mathrm{c} 1 \mathrm{~B}$, etc. These coefficients define the antenna beam tilt and pattern. Several sets of coefficients can be used simultaneously for several communication standards such as $2 \mathrm{G}, 3 \mathrm{G}$ and $4 \mathrm{G}$ standards. In addition, different coefficient sets can be used simultaneously for vertical sectorization. This technical term - also referred to as vertical cell splitting [2] - is used for the option to provide a small hot-spot network with a significant down-tilt and a macro cell network with more horizontal tilt over the same hardware. The antenna radiation patterns in Fig. 1 indicate that not only antenna beam tilt can be defined independently for two such "vertical sectors", but also the radiation pattern shapes are independently tunable. In the example of Fig. 1, the stronger downtilted patterns "RX1" and "TX1" are more optimized for upper side-lobe suppression than those of more horizontally tilted patterns "RX2" and TX2", to minimize interference between these two "vertical sectors". Other applications of multiple 


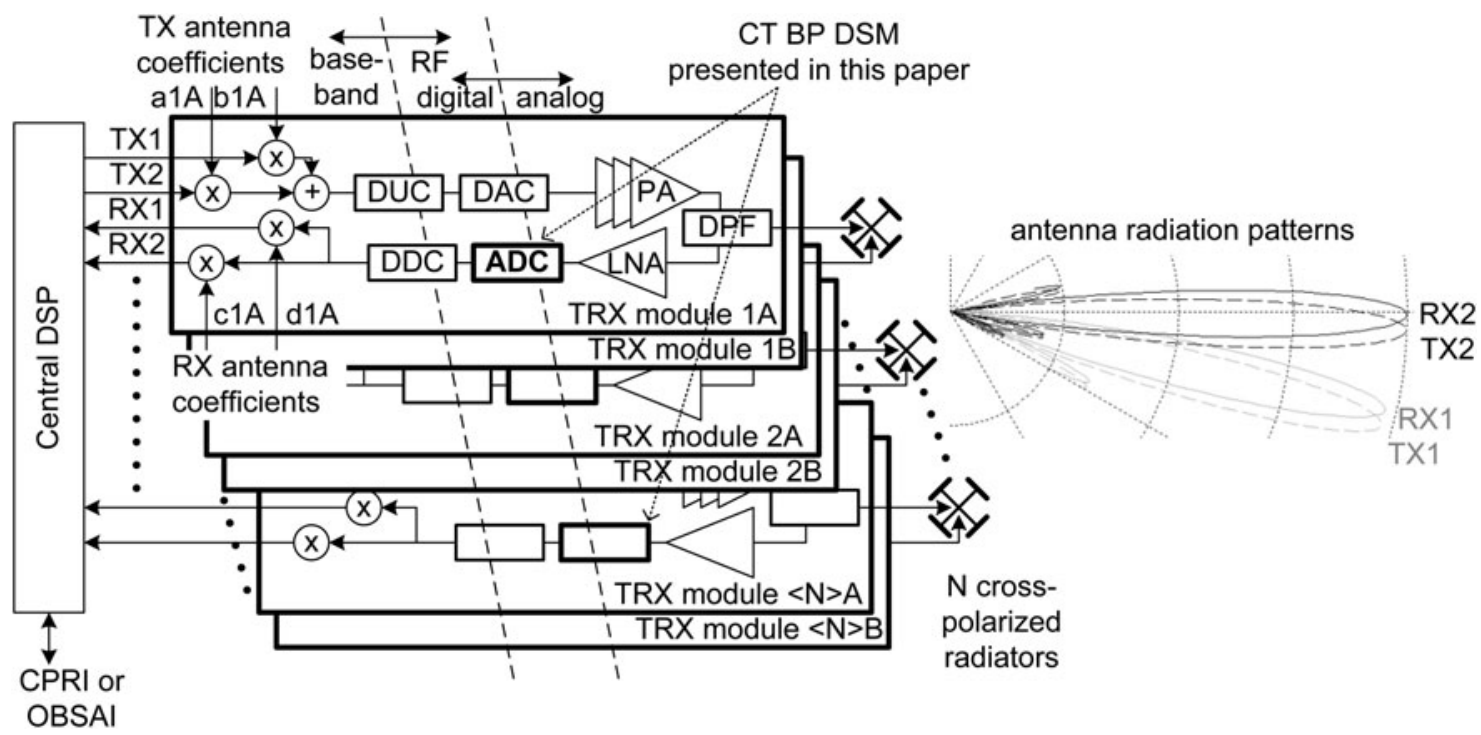

Fig. 1. AAS block diagram, showing the location of the CT BP DSM of this paper inside the modular AAS structure.

simultaneous antenna radiation patterns are diversity reception, multiple input, multiple output (MIMO), and RAN (radio access network) sharing (more than one network operator sharing the same RF hardware).

The transition from traditional base stations to AAS is a disruptive change in the system architecture. A similarly disruptive transition is carried out in transceiver design. For the AAS presented in Fig. 1, in both directions, RX and TX, conversion between analog and digital is carried out in the RF band at sampling rates of a few GS/s. Consequently, the up and down conversion from RF to baseband and vice versa is carried out digitally. This is a significant step toward a software-defined radio. In the RX path, one way to realize RF analog to digital conversion is to employ CT BP DSMs, as presented in this paper.

Requirements for AAS receivers differ from those for handset receivers and from those for conventional base-station receivers. Owing to the large number of receivers per antenna (typically $10-16$, i.e. 5-8 per polarization), cost targets are much lower than for conventional base station receivers. High-resolution analog to digital converter (ADCs) with several hundred $\mathrm{MHz}$ sampling rate plus off-chip image reject filters are not a commercially viable option in this case. On the other hand, performance requirements, especially image rejection and the absence of other spurs or inter-modulation products, are as stringent as for conventional base stations. Only the signal to noise ratio (SNR) requirement is slightly relaxed, since SNR increases by $7-9 \mathrm{~dB}$ when digitally combining 5-8 receive signals for each polarization. Unlike mobile receivers, in the base station case the entire bandwidth of a communications band needs to be received simultaneously. The presented receiver is designed for simultaneous reception of $3 \mathrm{GPP}$ bands 13 and 14 used for long term evolution (LTE) in the USA, with uplink frequencies ranging from 777 to $798 \mathrm{MHz}$.

\section{B) Circuit design: CT BP DSM versus zero-intermediate frequency (IF) receivers}

The CT BP DSM approach has been chosen for this receiver. The most important advantage of this receiver type is the absence of a local oscillator (LO). The RF signal is directly digitized without down-conversion to an IF or to baseband (zero IF). Not having an LO yields several benefits, as none of the challenges of zero-IF receiver design apply to CT BP DSM design:

(a) There is no LO leakage back to the antenna. For cellular infrastructure base-stations, there are stringent requirements on such in-band spurious emissions.

(b) No second-order inter-modulation (i.e. varying-envelope blockers causing second-order products centered around DC in baseband) is observed in CT BP DSMs.

(c) Zero-IF receivers frequently require significant effort on DC-offset cancellation, often involving control loops. CT BP DSMs do not suffer from DC offsets, since downconversion is performed digitally.

(d) Zero-IF receivers, even if applying digital correction or calibration techniques, would typically not reach an image rejection of $>70 \mathrm{~dB}$ as needed in base station receivers. With CT BP DSMs, there is no image reception.

Also, from a cost perspective, the CT BP DSM approach is appealing: there is no need for circuitry like mixers, quadrature LO generators, image reject filters, and conventional (low-pass) analog-to-digital-converters. A single chip directly digitizes the analog RF signal.

Challenges in RF CT BP DSM design are high linearity of the feedback DAC and of the transconductance and Q-enhancement stages. Significant attention also needs to be attributed to the LC resonators. They do not only need to have a high-quality factor; resonance frequency and Q-enhancement also need to be kept constant over PVT (process, voltage, temperature) corners.

Numerous RF CT BP DSMs have been reported over the last 15 years, e.g. [4-10], most of them using SiGe HBT technology and a single-bit quantizer. Only one of them [4] achieves SNDR and $B W$ suitable for AAS applications, but at a sampling rate of $40 \mathrm{~Gb} / \mathrm{s}$, making decimation filtering challenging.

The target of the design presented here was to increase the state-of-the-art in SiGe RF BP DSMs, while keeping the sampling rate below $4 \mathrm{GS} / \mathrm{s}$ in order to allow for simple, lowpower complementary metal oxide semiconductor (CMOS) decimation filtering. 


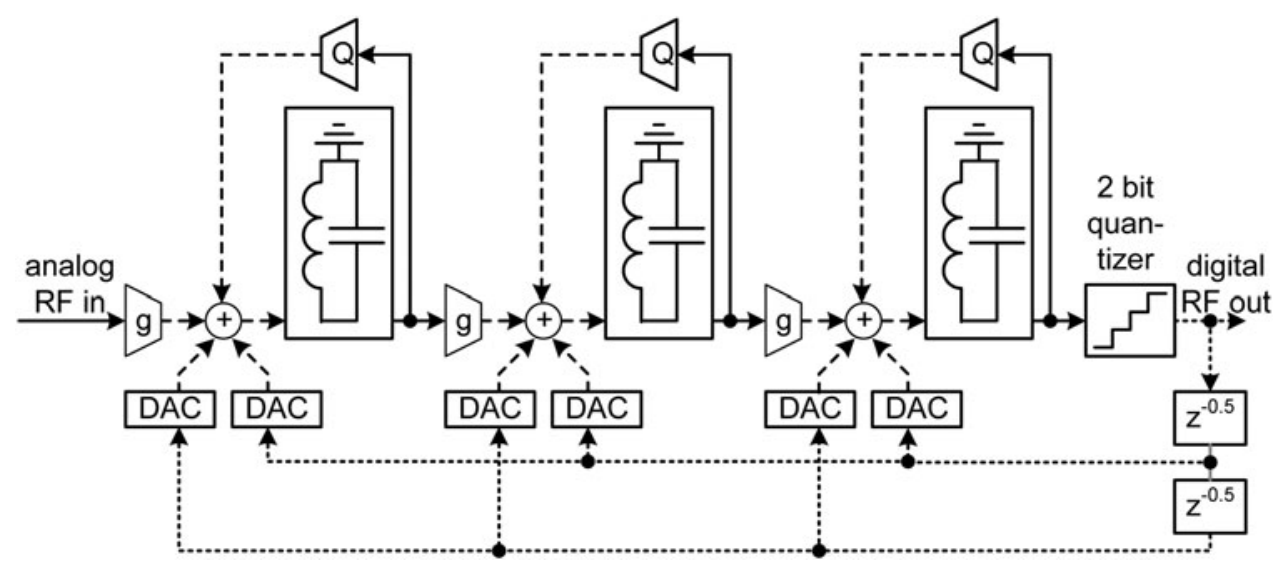

Fig. 2. Signal flow diagram of the realized 6th order CT BP DSM. Solid lines: voltage signals; dashed: current signals; dotted: 2-bit digital signals.

\section{DESIGN}

The signal flow diagram of the CT BP DSM is shown in Fig. 2. The topology is similar to [8], but sixth order and using a non-return-to-zero (NRZ) feedback. For clarity, the three signal types are drawn in different line styles: solid lines for voltage signals, dashed lines for current signals, and dotted lines for digital signals. The sixth order has been chosen because lab experiments with the CT BP DSM of [5], configured for fourth, sixth, and eighth order, yielded best RF performance when configured for sixth order. Quality factor of differential LC resonators is boosted by negative gm Q-enhancement circuits labeled "Q", as proposed in e.g. [5, 8].

Unlike [5], feedback from the digital output to each of the LC resonators is applied by two differently delayed DACs. This method has been proposed by [11] as "excessive loop delay compensation". This name implies that a discrete time DSM is designed and then translated into a CT DSM, and that any delay in the feedback path, other than the wanted $0.5 \mathrm{~T}$ or 1.0 $\mathrm{T}$ delay, causes a difference between the DT reference model and CT implementation. We did not take this design approach, but designed the CT DSM directly using the same methodology as in [5]. Nevertheless, the six feedback paths - two feedback DACs for each of the three resonators are required in order to have six degrees of freedom to independently tune all six coefficients of the numerator of the sixth order noise transfer function.

Unlike [11], this CT BP DSM uses NRZ feedback. Employing NRZ feedback increases the risk of inter-symbol interference: asymmetry in the differential DACs can cause a $2 \mathrm{~T}$ long pulse to have a charge different from twice the charge of a $1 \mathrm{~T}$ pulse, $\mathrm{T}$ being the clock period. On the other hand, using NRZ feedback reduces the speed requirement of the DACs by a factor of 2. Also, as has been calculated and proven by [12], impact of jitter on SNR is $9.3 \mathrm{~dB}$ lower for NRZ compared to RF feedback. We therefore decided to implement NRZ feedback.

One difference to $[4-10,13]$ is the use of two-bit (fourlevel) internal quantization. More than single-bit (two-level) quantization introduces the risk of mismatch between current sources, leading to non-linearity of the DAC. Such DAC non-linearity, when driven with a wideband quantization noise signal, leads to a flat noise floor filling the noise notch. In this design, we yet decide for four-level quantization and ensured sufficient DAC linearity by physical size (area) of current source resistors " $R$ " and " $2 R$ " in Fig. 3, the feedback $\mathrm{DAC}$ schematic. By performing Monte-Carlo mismatch simulations, we verified that spectral shaping of the error introduced by DAC-non-linearity, such as dynamic element matching, or digital correction algorithms as in [14] are not needed. HBTs Q1 and Q2 provide a reference voltage with negative temperature coefficient to current source transistors Q3 and Q4. Cascode transistors Q5 and Q6 are only needed to limit the collector voltage of $\mathrm{Q}_{3}$ and $\mathrm{Q} 4$, protecting them from breakdown. Q11, Q12 and Q13, Q14 steer the MSB and LSB currents to the positive or negative side of the LC resonator. They are driven with differential digital signals from the quantizer, where "MSB" and "LSB" denote the inverted signals. Q7-Q10 perform first-order compensation of base to collector crosstalk of Q11-Q14.

The top-level layout is shown in Fig. 4. Top metal is displayed in black, all other metal layers in different shades of gray. The three identical inductors of the three LC resonators can be recognized. The two smaller inductors are inductive degeneration of the RF input stage. The quantizer and the feedback DACs are placed close to the centre in order to keep the routing of high-speed digital signals as short as possible.

\section{MEAsUREMENTRESULTS}

The $2.3 \mathrm{~mm} \times 2.6 \mathrm{~mm} \mathrm{SiGe} \mathrm{BiCMOS}$ die shown in Fig. 4 has been assembled into a standard $6 \mathrm{~mm} \times 6 \mathrm{~mm}$ QFN plastic

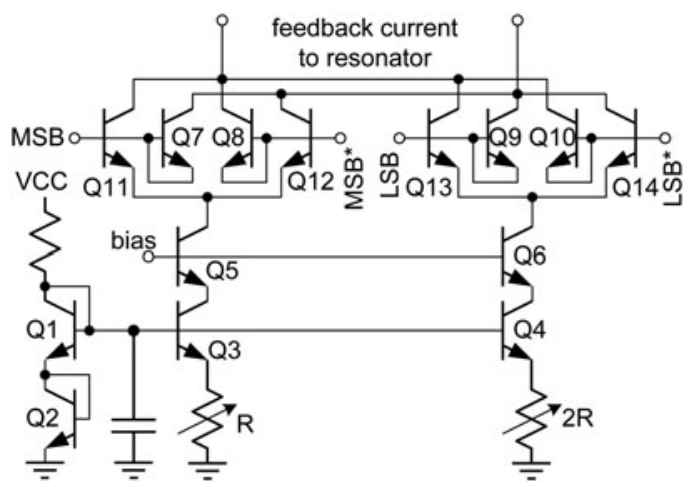

Fig. 3. Schematic diagram of one of the 2-bit feedback DACs. 


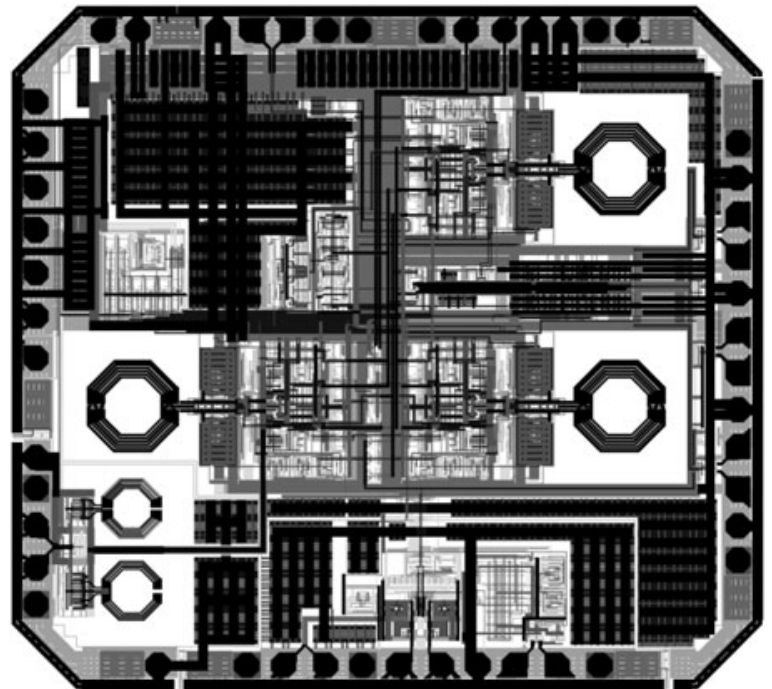

Fig. 4. Layout of the $2.3 \mathrm{~mm} \times 2.6 \mathrm{~mm} \mathrm{SiGe}$ BiCMOS die. Top metal is black, other metals gray.

package and mounted on a standard FR4 epoxy printed circuit board (PCB).

For the FFT spectrum of Fig. 5, a single-tone CW test signal of $-30 \mathrm{dBm}$ at $774 \mathrm{MHz}$ has been applied to the RF input. The $3.134 \mathrm{GS} / \mathrm{s}$ digital output data were captured, and an FFT was calculated offline. FFT length as shown in Fig. 5 was 280000 symbols, resulting in an FFT frequency bin spacing of $11 \mathrm{kHz}$. The peak of the FFT spectrum is $-15.15 \mathrm{~dB}_{\mathrm{FS}}$ ( $\mathrm{dB}$ full scale). Owing to Hann windowing, the signal spreads over $2-3$ of the $11 \mathrm{kHz}$ frequency bins, so that the total signal power is $\sim 3 \mathrm{~dB}$ higher $\left(-12 \mathrm{~dB}_{\mathrm{FS}}\right)$.

The only spur seen over the $100 \mathrm{MHz}$ measurement bandwidth from 740 to $840 \mathrm{MHz}$ is at $-79 \mathrm{~dB}_{\mathrm{FS}}=-64 \mathrm{dBc}$ l $812 \mathrm{MHz}$. This frequency is an alias of the third harmonic of the RF input frequency, sampled by the quantizer. It appears at a frequency of $812 \mathrm{MHz}$ :

$$
f_{3 \text { rd harmonic alias }}=f_{\text {clock }}-3 \times f_{\text {signal }}
$$

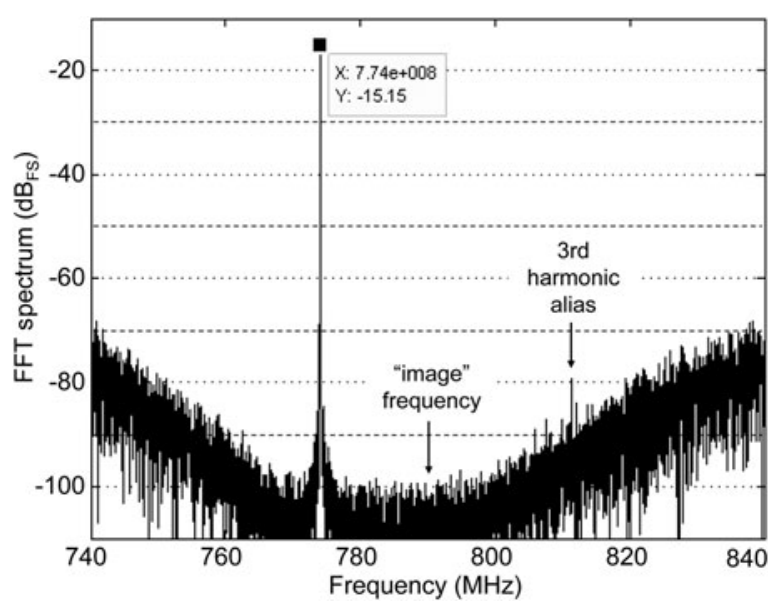

Fig. 5. FFT spectrum of output data stream with CW RF input test signal.

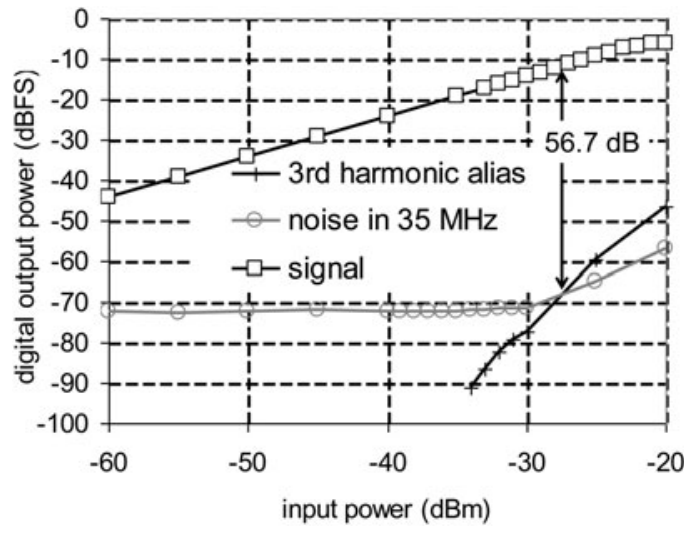

Fig. 6. Signal, noise, and distortion power versus RF input power. Squares: wanted RF signal; circles: noise integrated in $35 \mathrm{MHz}$; "+ ": alias of third harmonic. Arrows indicate SNDR and SNR in $35 \mathrm{MHz}$ bandwidth.

In addition to this third harmonic, in [13] we reported a first-order "image" reception at

$$
f_{\text {image }}=\frac{1}{2} f_{\text {clock }}-f_{\text {signal }}
$$

This image was caused by a divided clock signal at $1.567 \mathrm{GHz}$ being applied to an output buffer of the chip, and from there coupling into the DSM. If a sub-harmonic at half the clock frequency is coupling into e.g. the clock tree, in time domain, every other bit (e.g. the in-phase bits) has a slightly different clock period than the other bits in between (the quadrature bits " $Q$ "). This leads to charge being fed back by the feedback DACs being different between I and Q clock cycles. In-phase bits being treated differently from quadrature bits results in image reception in any receiver. By avoiding this sub-harmonic coupling mechanism, we no longer observe an image in the $83 \mathrm{~dB}$ measurement dynamic range from -15 to $-98 \mathrm{~dB}_{\mathrm{FS}}$.

Results of an input power sweep are shown in Fig. 6 . The $x$-axis is $\mathrm{CW} \mathrm{RF}$ input power in $\mathrm{dBm}$ and $y$-axis is digital output power in $\mathrm{dB}_{\mathrm{FS}}$. Wanted signal power is marked by squares. $\mathrm{P}_{1} \mathrm{~dB}$ compression point is approximately at $-22 \mathrm{dBm}$ input, and $-7 \mathrm{dBFS}$ output power. As would be expected, slope of the alias of the third harmonic (marked

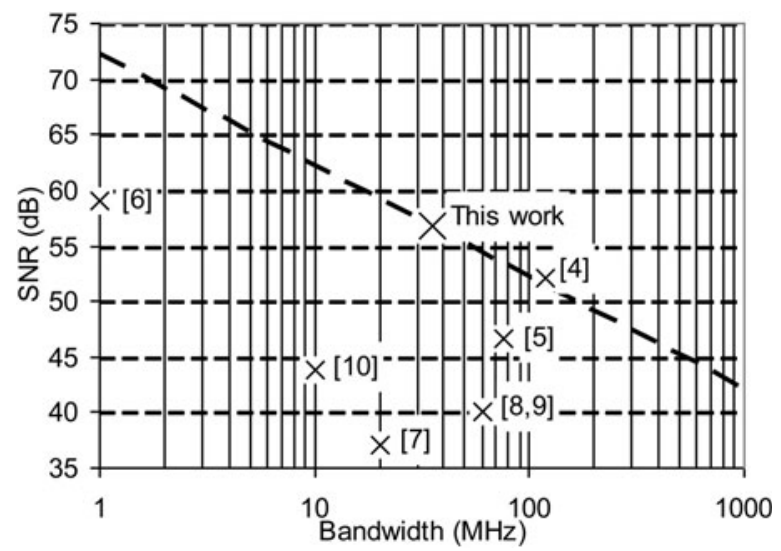

Fig. 7. SNR of previously published RF CT BP DSMs versus bandwidth, in comparison to this work. The dashed line is a line of constant SNR of $72.1 \mathrm{~dB}$ normalized to $1 \mathrm{MHz}$ bandwidth. 
Table 1. Performance comparison.

\begin{tabular}{|c|c|c|c|c|c|c|c|c|}
\hline & [4] & {$[5]$} & [6] & [7] & {$[8]$} & [9] & {$[10]$} & This work \\
\hline $\mathrm{RF}(\mathrm{GHz})$ & 2 & 0.9 & 0.95 & 1 & 2.2 & 2.4 & 0.9 & 0.78 \\
\hline Clock freq. (GHz) & 40 & 3.6 & 3.8 & 4 & 7.5 & 3 & 2.2 & 3.13 \\
\hline$B W(\mathrm{MHz})$ & 120 & 75 & 1 & 20 & 60 & 60 & 10 & 35 \\
\hline SNR (dB) & 52 & 46.6 & 59 & 37 & 40 & 40 & 43.8 & 56.7 \\
\hline SNR in $1 \mathrm{MHz}(\mathrm{dB})$ & 72.8 & 65.4 & 59.0 & 50.0 & 57.8 & 57.8 & 53.8 & 72.1 \\
\hline DC power $(\mathrm{W})$ & 1.6 & 0.9 & 0.075 & 0.45 & 0.45 & 0.04 & - & 1.2 \\
\hline
\end{tabular}

with " + ") is $3 \mathrm{~dB} / \mathrm{dB}$. A trace showing noise power integrated over the full bandwidth of $35 \mathrm{MHz}$ is marked with circles. SNR and SNDR at $-27.5 \mathrm{dBm}$ (see arrow in Fig. 6) in $35 \mathrm{MHz}$ bandwidth are $56.7 \mathrm{~dB}$ and $53.7 \mathrm{~dB}$, respectively.

Two-tone tests have also been conducted. Unlike in [8], third-order inter-modulation $\left(\mathrm{IM}_{3}\right)$ products scale with $3 \mathrm{~dB} /$ $\mathrm{dB}$ as with "normal" analog receive circuits, e.g. amplifiers and mixers. Input referred third-order intercept point is $-6.6 \mathrm{dBm}$. Typical noise figure at room temperature is $10 \mathrm{~dB}$. Two-tone spurious-free dynamic range (SFDR) as defined by

$$
\begin{aligned}
S F D R_{\mathrm{dB}}= & \frac{2}{3}\left(I I P_{3 \mathrm{dBm}}-P_{\text {noise }}\right) \\
= & \frac{2}{3}\left(I I P_{3 \mathrm{dBm}}-N F-10 \log _{10}\left(\frac{B W}{\mathrm{~Hz}}\right)\right. \\
& -174 \mathrm{dBm} / \mathrm{Hz})
\end{aligned}
$$

is $54.6 \mathrm{~dB}$ in $35 \mathrm{MHz}$ and $64.9 \mathrm{~dB}$ in $1 \mathrm{MHz}$ bandwidth. This is an improvement of $9 \mathrm{~dB}$ over [5].

Figure 7 and Table 1 compare the performance of this circuit to previously published CT BP DSMs. The plot displays SNR versus bandwidth for [4-10]. The dashed line indicates a line of constant SNR normalized to $1 \mathrm{MHz}$ bandwidth of $72.1 \mathrm{~dB}$. Excluding [4] with an exotic sampling rate of $40 \mathrm{GS} / \mathrm{s}$, all other referenced CT BP DSMs have at least $6.7 \mathrm{~dB}$ lower SNR in $1 \mathrm{MHz}$.

A different application of RF CT BP DSMs is conversion from RF analog to high-speed digital for driving switch mode "class-S" power amplifiers, as proposed e.g. by $[5,8$, 10]. For this purpose, a W-CDMA RF downlink signal has

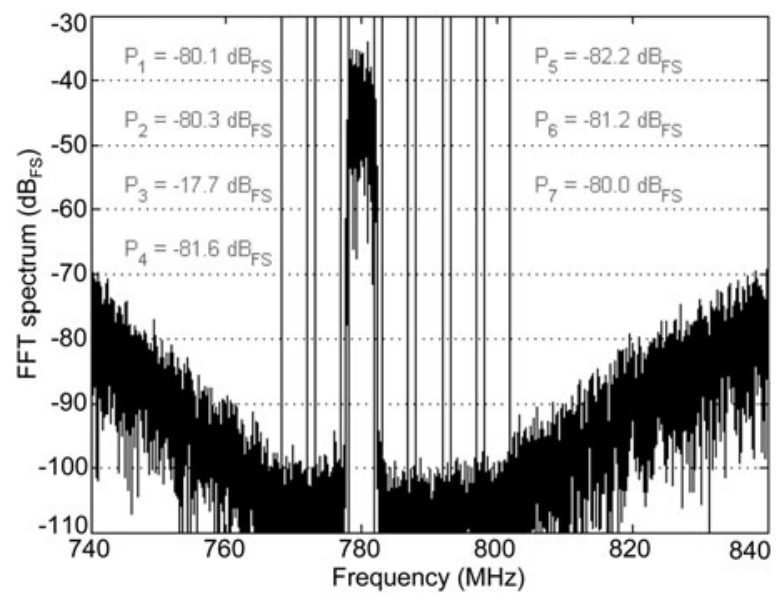

Fig. 8. FFT output spectrum obtained with a single-carrier W-CDMA RF input signal. been applied to the DSM of this paper. The corresponding FFT output spectrum is shown in Fig. 8. For seven 3.84 MHz channels with $5 \mathrm{MHz}$ spacing, spanning over the $35 \mathrm{MHz}$ wide noise notch of the DSM, the integrated rms power is calculated and displayed in gray in Fig. 8. It is seen that the $\mathrm{W}$-CDMA signal power in channel 3 is $-17.7 \mathrm{~dB}_{\mathrm{FS}}$, while power in all other channels is below $-80 \mathrm{~dB}_{\mathrm{Fs}}$. ACLR (adjacent power leakage ratio) is $-62.6 \mathrm{dBc}$. This is an 8.2 dB improvement over [5].

Also, a multi-carrier W-CDMA downlink signal has been applied to this CT BP DSM. To demonstrate the instantaneous bandwidth, three carriers have been spread over the $35 \mathrm{MHz}$ wide noise notch. Figure 9 shows the FFT spectrum of this measurement. FFT signal power in channels 1, 2, and 7 at the digital output is $-\mathbf{2 4 . 3}$ to $-\mathbf{2 4 . 2} \mathrm{dB}_{\mathrm{FS}}$ per carrier, $-19.5 \mathrm{~dB}_{\mathrm{FS}}$ in total. At this relatively low power level, only two of the four possible digital symbols occur in the output data stream. Hence, a single-bit class-S PA, which is much easier to realize than two-bit, is sufficient. Adjacent channel power in channels 3 and 6 is $-76.4 \mathrm{~dB}_{\mathrm{FS}}$, so three-carrier ACLR is $-52.1 \mathrm{dBc}$, leaving a $7 \mathrm{~dB}$ margin to the $3 \mathrm{GPP}$ base station requirement of $-45 \mathrm{dBc}$, allowing for some adjacent channel leakage ratio (ACLR) degradation by class-S power amplifier non-linearity. To our knowledge, this is the first multicarrier downlink spectrum published for any RF CT BP DSM, and possibly the first CT BP DSM suitable for this application.

\section{CDNCLUSION}

After providing an introduction to AAS, an RF CT BP DSM receiver used in such an AAS has been presented.

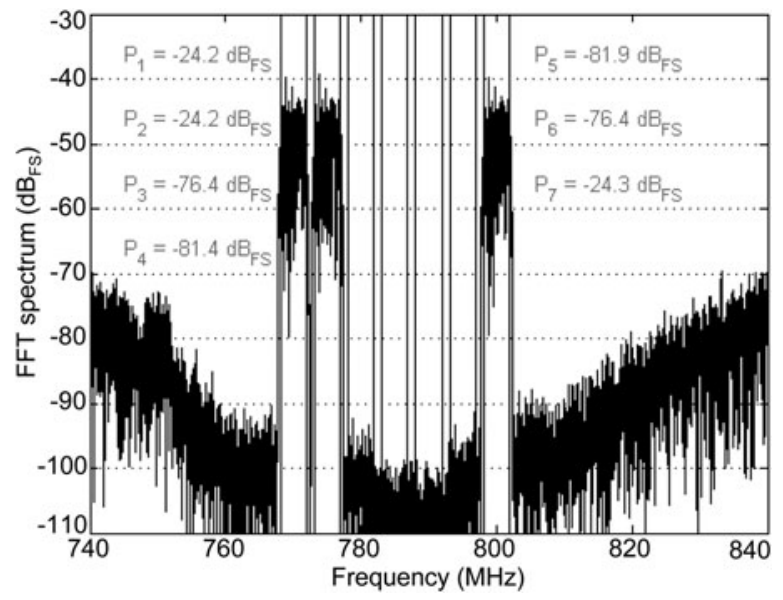

Fig. 9. FFT output spectrum obtained with a three carrier W-CDMA RF input signal. 
Employing sixth-order noise shaping and 2 bit internal quantization, it achieves an SNR of $56.7 \mathrm{~dB}$ in $35 \mathrm{MHz}$ bandwidth, at a moderate sampling frequency of $3.1 \mathrm{GHz}$. To our knowledge, this is the first RF BP DSM receiver suitable for use in cellular base-station AAS, with a $6.7 \mathrm{~dB}$ SNR improvement over comparable previously published designs. This is a breakthrough in AAS receiver design, since this single RF-digitizing IC replaces quadrature LO generator, IQ-down-converter, image reject filter, and two (I and Q) high-resolution baseband ADCs. Also, suitability of this CT BP DSM as a modulator for class-S power amplifiers has been demonstrated, with a $7 \mathrm{~dB}$ improvement in ACLR over previously published modulators.

\section{REFERENCES}

[1] Linehan, K.; Chandrasekaran, R.: Active Antennas: The Next Step in Radio and Antenna Evolution. 2011 [Online]. Available at http:// docs.commscope.com/Public/active_antenna_system_white_paper. pdf

[2] Nokia Siemens Networks. Active Antenna Systems - A step-change in base station site performance. 2012 [Online]. Available at http:// www.nokiasiemensnetworks.com/sites/default/files/document/ nokia_siemens_networks_active_antenna_system_white_paper_26_01_12_0.pdf

[3] Karthaus, U.; Sukumaran, D.; Schmidt, L.; Ahles, S.; Wagner, H.: A $45 \mathrm{dBm}$ balanced Power Amplifier Module based on four fully integrated Doherty PA MMICs, in European Microwave Conference, Amsterdam, 2012, 1027-1030.

[4] Chalvatzis, A.; Gagnon, E.; Repeta, M.; Voinigescu, S.: A low-noise $40 \mathrm{GS} / \mathrm{s}$ continuous-time bandpass $\triangle \Sigma$ ADC centered at $2 \mathrm{GHz}$ for direct sampling receivers. IEEE J. Solid-State Circuits, 42 (2007), 1065-1074.

[5] Karthaus, U.; Ahles, S.; Elmaghraby, A.; Schick, C.; Datta, P.K.; Rheinfelder, C.: A $900 \mathrm{MHz}, 3.6 \mathrm{~Gb} / \mathrm{s}$ bandpass DSM receiver with $55.7 \mathrm{~dB}$ two-tone SFDR in $1 \mathrm{MHz}$ bandwidth, in IEEE Int. Microwave Symp., San Francisco, 2009, 1133-1136.

[6] Thandri, B.; Silva-Martinez, J.: A $63 \mathrm{~dB} 75-\mathrm{mW}$ band-pass RF ADC at $950 \mathrm{MHz}$ using $3.8-\mathrm{GHz}$ clock in $0.25-\mu \mathrm{m}$ SiGe BiCMOS technology. IEEE J. Solid-State Circuits, 42 (2007), 269-279.

[7] Cherry, J.; Snelgrove, W.; Gao, W.: On the design of a fourth-order continuous-time LC delta-sigma modulator for UHF A/D conversion. IEEE Trans. Circuits Syst. II, 47 (2000), 518-530.

[8] Schmidt, M. et al.: Continuous-time bandpass delta-sigma modulator for a signal frequency of $2.2 \mathrm{GHz}$, in German Microwave Conf., Munich, 2009, 1-4.

[9] Ryckaert, J. et al.: A $2.4 \mathrm{GHz}$ low-power sixth-order RF bandpass delta sigma converter in CMOS. IEEE J. Solid-State Circuits, 44 (2009), 2873-2880.

[10] Scheytt, J.C.; Ostrovskyy, P.; Gustat, H.: RF bandpass delta-sigma modulators for highly-efficient class-S transmitters in SiGe BiCMOS technology, in IEEE Int. Conf. Wireless Information Technology and Systems, Honolulu, 2010, 1-4.

[11] Gao, W.; Shoaei, O.; Snelgrove, W.M.: Excess loop delay effects in continuous-time delta-sigma modulators and the compensation solution, in IEEE Int. Symp. Circuits and Systems, Hong Kong, 1997, 65-68.

[12] Cherry, J.A.; Snelgrove, W.M.: Clock jitter and quantizer metastability in continuous-time delta-sigma modulators. IEEE Trans. Circuits Syst. II, 46 (1999), 661-676.
[13] Karthaus, U.; Ahles, A.; Elmaghraby, A.; Wagner, H.: A 2-bit, 3.1 GS/s, band-pass DSM receiver with $53.7 \mathrm{~dB}$ SNDR in $35 \mathrm{MHz}$ bandwidth, in European Microwave Conf., Amsterdam, 2012, 661-676.

[14] Kauffman, J.G.; Witte, P.; Becker, J.; Ortmanns, M.: An $8.5 \mathrm{~mW}$ continuous-time delta sigma modulator With $25 \mathrm{MHz}$ bandwidth using digital background DAC linearization to achieve $63.5 \mathrm{~dB}$ SNDR and $81 \mathrm{~dB}$ SFDR. IEEE J. Solid-State Circuits, 46 (2011), 2869-2881.

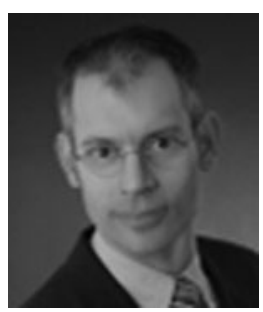

Udo Karthaus received his "Dipl.-Ing." and Ph.D. degrees in Electrical Engineering from the University of Paderborn, Germany in 1995 and 2000, respectively. Thereafter, he worked for 6 years at Atmel, designing SiGe RFICs for base-station and handset applications, as well as mixed signal CMOS circuits. In 2006, he joined Ubidyne, where he built and led the SiGe RF design team. Since 2008, he has been leading Ubidyne's power amplifier MMIC development. He has authored more than 20 technical papers and 20 patent applications. His pioneer UHF RFID paper in IEEE JSSC has been referenced more than 160 times.

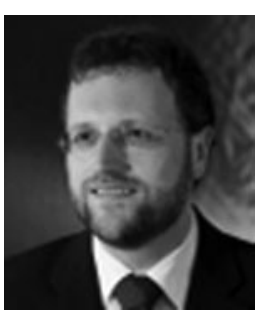

Stephan Ahles received his M.Sc. degree in 1996 from University of Southampton (UK). He then worked for 10 years in various development engineering roles specializing on RF transceiver ICs, before joining Ubidyne in 2006 as Senior RFIC Design Engineer where he contributed to the development of Ubidyne's chipset.

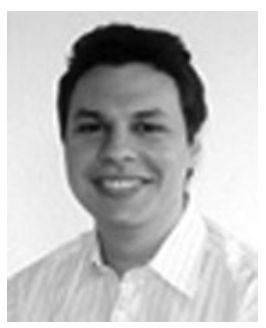

Ahmed Elmaghraby received the B.Sc. degree in electronics and communication engineering from Ain Shams University, Cairo, Egypt in 2003. In 2007, he received the M.Sc. degree in communication technology from the University of Ulm, Germany. After that, he joined Ubidyne $\mathrm{GmbH}$ for more than 4 years where he worked on $\mathrm{RF} / \mathrm{analog}$ circuit design. He is currently pursuing the Ph.D. degree at the University of Erlangen-Nuremberg. His research interests are focused on CMOS radio receivers.

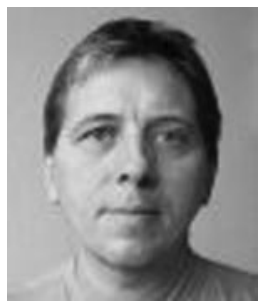

Horst Wagner received his Ph.D. in Physics from the Technical University of Vienna, Austria in 1999. He has since worked on circuit design of highspeed microprocessors at Compaq, Hewlett Packard, and Intel in Massachusetts. He is currently heading IC development at Ubidyne in Ulm, Germany with focus on state-of-the-art RF frequency mixed signal circuits and delta-sigma modulators for use in active antenna systems. 